\title{
Insieme è meglio. Riflessioni pedagogiche sul caso "Scarabò. Una città per educare"
}

Together is better. Pedagogical reflections on the case

"Scarabò. A city to educate"

Fabrizio d'Aniello*

\section{Riassunto}

Questo articolo presenta solo alcuni risultati emersi da una complessa attività di ricerca sul campo effettuata tra il 2018 e il 2019. La ricerca è stata motivata dalla partecipazione al progetto internazionale denominato Trans-Urban EU-China, dedicato alla «transizione verso la sostenibilità urbana attraverso città socialmente integrative nell'Ue e in Cina». Nello specifico, lo studio empirico si è concentrato su "Scarabò. Una città per educare", festival dell'educazione che si svolge ogni anno nel centro storico di Macerata, mettendo a disposizione dei bambini e di tutta la cittadinanza più di 40 laboratori educativi e varie attività ludiche. In questa occasione, vengono riportati i risultati delle interviste somministrate a 111 bambini partecipanti all'iniziativa e ci si sofferma pedagogicamente sui temi della città educante, colta secondo la prospettiva dell'educazione permanente, e degli spazi urbani come spazi di relazione significativa. "Insieme è meglio" è l'espressione che riassume le risposte dei bambini, le quali invitano a ripensare educativamente i luoghi della città.

Parole chiave: città educante, educazione permanente, spazi urbani, bambini e socialità urbana, "Scarabò. Una città per educare", Trans-Urban EU-China.

\section{Abstract}

This article presents only some results that emerged from a complex field research activity carried out between 2018 and 2019. The research was motivated by the participation in the "Trans-Urban EU-China" international project, dedicated to the «transition towards urban sustainability through socially integrative cities in the EU and in China». Specifically, the empirical study focused on "Scarabò. A city to educate", a festival of education that takes place every year in the historic center of Macerata, making more than 40 educational workshops and several recreational activities available to children and all citizens. On this occasion, the outcomes of the interviews given by 111 children participating in the initiative are reported, and we pedagogically dwell

\footnotetext{
${ }^{*}$ Professore associato di Pedagogia generale e sociale presso l'Università degli Studi di Macerata. E-mail: fabrizio.daniello@unimc.it.
}

Doi: 10.3280/ess2-2020oa9391 
on the subjects of the educating city, seen from the perspective of lifelong education, and of urban spaces conceived as spaces of significant relationship. "Together is better" is the expression that summarizes the children's responses, which urge to rethink the places of the city educationally.

Key-words: educating city, lifelong education, urban spaces, children and urban social relations, "Scarabò. A city to educate", Trans-Urban EU-China.

Articolo sottomesso: 18/03/2020, accettato 2/04/2020

\section{Introduzione: il quadro di partenza e Scarabò}

Questo articolo si propone di presentare alcuni risultati emersi da una complessa attività di ricerca sul campo svolta tra il 2018 e il 2019, sollecitata dalla partecipazione, in qualità di research team member, al progetto denominato Trans-Urban EU-China. Tale progetto, finanziato dal programma Horizon 2020, è tuttora in corso e conta sul coinvolgimento di numerosi partner, di matrice universitaria e non, situati in Austria, Belgio, Cina, Germania, Israele, Italia e Norvegia. Il progetto, altresì, concerne esattamente la «transizione verso la sostenibilità urbana attraverso città socialmente integrative nell'Ue e in Cina». Il contributo fornito dalla maggioranza dei partner è perlopiù tecnicotecnologico, architettonico-urbanistico, ingegneristico, geo-politico e di ecologia urbana, mentre la dimensione pedagogica di studio è demandata all'area education dell'unità locale dell'Università degli Studi di Macerata, a sua volta "parte terza" associata al Politecnico di Torino e impegnata anche sul versante filosofico e dell'agricoltura urbana. Il task assegnato a detta area, in collaborazione con la Chinese Academy of Science and Technology for Development (Casted), riguarda il potenziale incremento di inclusione sociale urbana tramite l'educazione e l'innovazione tecnologica e, più specificatamente, ha a che fare con: il ruolo della continuing education, delle reti educative e degli strumenti digitali per realizzare quartieri e città socialmente integrativi; l'influenza della continuing education sul tessuto sociale urbano; la funzione della continuing education come servizio primario per una società basata sulla conoscenza e come meccanismo fondamentale per promuovere armonia e unità; il rapporto tra politiche formative, invecchiamento attivo e politiche di urbanizzazione. Obiettivo generale, quindi, è quello di rilevare e rafforzare il concorso dell'educazione e della tecnologia in ordine ad uno sviluppo urbano sostenibile, capace di includere e soddisfare bisogni di crescita e realizzazione di diverse persone.

Entro questo quadro complessivo di riferimento, andando ad affiancare le riflessioni dei colleghi pedagogisti sul co-housing e l'invecchiamento attivo, 
sulla configurazione pedagogica degli spazi scolastici e i musei della scuola, mi sono dedicato a contributi teorici sull'idea-guida di educazione permanente, sul concetto di città educante e sulla stringente interazione tra i due temi. Parallelamente, ho avuto la possibilità di concentrare empiricamente lo studio sul festival dell'educazione intitolato "Scarabò. Una città per educare".

Scarabò, dal 2017, ogni anno e prevalentemente nel penultimo fine settimana di maggio, si svolge nelle strade, nelle piazze, nelle corti interne di palazzi antichi e in altri spazi pubblici e privati, soprattutto esterni, del centro storico di Macerata, dando vita a più di 40 laboratori educativi e formativi (gestiti da educatori, formatori, docenti, volontari, artigiani, commercianti del territorio, liberi professionisti e artisti) rivolti a bambini e bambine dai 3 ai 13 anni, nonché alla cittadinanza tutta, accogliendo peraltro persone di paesi e città limitrofi e non solo. Esemplificando, siffatti laboratori spaziano: dall'educazione alimentare all'attività giornalistica, dalla conoscenza/manipolazione dei colori alla lettura animata e comprensione di brani mitologici adattati, dall'introduzione all'opera lirica alla presentazione della storia e della cultura albanese, dall'intreccio e la tintura artigianali alla danza contemporanea, dalla manualità creativa alla pigmentazione naturale, dalla tessitura a mano alla conoscenza della vita nello spazio, dal riciclo creativo alla motricità fine scortata dalla familiarizzazione con la lingua inglese, dall'educazione musicale alla produzione di origami, dal teatro alla danza popolare, ecc. Come detto, questi sono soltanto alcuni esempi dei laboratori attivati, a cui vanno aggiunte svariate attività ludiche e sportive.

A monte, la motivazione della scelta del "caso Scarabò" è stata dettata dagli intrecci indicativi con la tematica della città/comunità educante colta secondo una prospettiva di educazione permanente. In altri termini, la focalizzazione teorica su questo "oggetto" sembrava trovare un'idonea collocazione contestuale e pratico-educativa proprio in Scarabò, pur conscio della limitatezza temporale dell'iniziativa. A conferma verbale dell'ipotesi di partenza, nella fase preparatoria della ricerca, l'ideatrice e coordinatrice del festival, dottoressa Laura Copparoni, intervistata dal sottoscritto, ha così affermato:

«Una città che mette in campo un progetto educativo significativo concorre indubbiamente ad offrire ai suoi abitanti una vita migliore. Con ciò, la città diviene oggetto e spazio di educazione per alimentare se stessa attraverso la crescita delle persone che la abitano. Se pensiamo all'educazione come ad un compito di cui si fa carico un'intera comunità, come fatto collettivo e come pratica sociale, dovremmo pensare allo spazio cittadino come ad un ambiente in cui ciascuna persona può apportare il proprio contributo e nel quale ognuna può soddisfare la totalità dei propri bisogni educativi. In questo senso, la città diventa educativa, luogo di apprendimento e di educazione permanente». 
In aggiunta, la motivazione della scelta del "caso Scarabò", coerentemente con l'avallo dato dal coordinatore di progetto tedesco per la sua plausibile attinenza ai contenuti del task assegnato, è stata dettata dall'ipotetico ancoraggio dell'evento alle questioni delle reti educative, dell'integrazione sociale, dell'inclusione e dell'armonia/unità.

Tali plausibilità e ipotesi, dunque, sono state poste al vaglio della ricerca sul campo, la quale ha inteso approfondire da una prospettiva "ingenua" anche $\mathrm{i}$ medesimi costrutti di città/comunità educante e di educazione permanente, oltre ad argomenti inerenti il design urbano e il ruolo della tecnologia nell'"'apprendimento urbano". Così, nelle edizioni di Scarabò del 2018 e del 2019 sono stati intervistati 116 adulti, 111 bambini e 7 coordinatori dei laboratori. In questa sede, mi limiterò a considerare le risposte date dai bambini (associando a questo termine pure $\mathrm{i}$ fanciulli intervistati), dando conto di risultati parziali a fronte di un'indagine evidentemente più ampia e nondimeno esaustiva circa quanto addotto, che verrà esplicata con un lavoro monografico successivo.

\section{I campioni e l'attività di ricerca}

Innanzitutto, è doveroso precisare che, avendo ricorso ad una procedura di campionamento non probabilistico, i campioni di riferimento sono ovviamente non rappresentativi, pertanto le "conclusioni" a cui si perverrà non si prestano a generalizzazioni.

Ciò premesso, i 111 bambini sono suddivisibili in tre campioni: 53 tra bambini e bambine intervistati nell'edizione 2018 (19 e 20 maggio 2018) su 360 partecipanti, 35 intervistati in quella del 2019 (18 e 19 maggio 2019) su 288 partecipanti e 23 ancora in quella del 2019, ma intervistati in concomitanza con un disegno richiesto.

Il primo campione ha un range d'età compreso tra 4 e 12 anni, di cui 4 frequentanti la scuola dell'infanzia, 45 la scuola primaria, 3 la scuola secondaria primo grado e 1 privo di indicazione in merito. 18 sono i maschi, 35 le femmine. Questa, invece, la distribuzione geografica di residenza: 47 Macerata, 1 Civitanova Marche, 1 Corridonia, 1 Lido di Fermo, 1 Passo di Treia, 1 Montelupone, 1 Villa Potenza.

Il secondo campione ha un range d'età compreso tra 4 e 11 anni, di cui 3 frequentanti la scuola dell'infanzia e 32 la scuola primaria. 11 sono i maschi e 24 le femmine. Distribuzione geografica di residenza: 22 Macerata, 2 Morrovalle, 1 Passo di Treia, 1 Montelupone, 1 Monte San Giusto, 1 Porto Potenza Picena, 1 Borgo San Giovanni, 1 Casette Verdini, 1 Fermo, 1 Petriolo, 1 Senigallia, 1 Fano, 1 proveniente dalla Corsica. 
Il terzo campione ha un range d'età compreso tra 4 e 9 anni, di cui 5 frequentanti la scuola dell'infanzia e 18 la scuola primaria. I maschi sono 7, le femmine 16. Distribuzione geografica di residenza: 10 Macerata, 2 Montecassiano, 2 Montecosaro, 2 Castelraimondo, 1 Recanati, 1 Montelupone, 1 Petriolo e 4 privi di indicazioni in merito.

Il campione $\mathrm{n}$. 1 è stato sottoposto ad un'intervista inerente: 1 . la natura di Scarabò (percezione del che cosa sia); 2. l'attività che è piaciuta di più e le ragioni della preferenza; 3 . che cosa è stato appreso grazie all'evento; 4 . se è piaciuto o meno stare con altri e perché; 5 . se si vorrebbe la città sempre così $\mathrm{e}$ perché; 6 . come si vorrebbe la propria città, specie dal punto di vista degli spazi urbani e del loro utilizzo.

In seguito ai risultati di sintesi ottenuti con il campione n. 1, il campione $n$. 2 è stato sottoposto ad un'intervista avente lo scopo di sondare maggiormente le sensazioni esperite nella partecipazione ad attività con altri bambini e con adulti, conosciuti e sconosciuti.

Al campione n. 3, invece, sempre in linea con l'intenzione di avere contezza di un'idea peculiare di città e delle sensazioni legate allo stare insieme ad altri in questa stessa città, è stato chiesto di disegnare la propria città ideale e di disegnarsi mentre si gioca con altri in essa. Nel corso del disegno, l'addetto all'intervista si è fatto inoltre raccontare le scelte dei bambini, concentrandosi particolarmente sulla rappresentazione della città (sui motivi della sua rappresentazione) e sugli stati d'animo che potessero scaturire dal giocare in città con gli altri, sia in positivo sia in negativo.

A causa della somministrazione delle interviste, effettuata da 9 soggetti, le medesime seguono una sequenza strutturata, ad eccezione di quelle fatte in occasione dei disegni (seppur basandosi su una traccia comune), essendo l'oggetto naturalmente variabile. Tutte le interviste, concessa la liberatoria ad hoc da parte dei genitori, sono state audio-registrate e poi trascritte letteralmente in formato elettronico.

Le domande poste al campione $\mathrm{n}$. 1, infine, previa illustrazione delle finalità progettuali legate a Trans-Urban EU-China e conseguentemente degli item principali da mettere a fuoco, condivisi con la predetta area education, sono state concordate con la coordinatrice del festival a valle di un'estesa intervista da lei rilasciata in data 26 marzo 2018 al fine di comprendere: 1. la realtà di Scarabò; 2. i motivi della scelta del centro storico; 3 . il rapporto tra città, apprendimento ed educazione; 4. il significato di città educante; 5 . la relazione tra città educante ed educazione permanente; 6 . la relazione tra spazi fisici ed educazione; 7. i destinatari dell'iniziativa e le figure educative coinvolte; 8. le mete educative di Scarabò; 9. il rapporto tra Scarabò e inclusione sociale; 10. il rapporto tra Scarabò e tecnologia. 
Anche la domanda posta al campione n. 2 e l'attività richiesta al campione n. 3, unitamente alla narrazione corrispondente, sono state condivise con l'area education e concordate con la coordinatrice di Scarabò.

\section{I risultati della ricerca}

Partendo dal campione n. 1, alla domanda n. 1 "Che cosa è Scarabò?", diretta a rilevare lo scarto percettivo e di vissuto tra una situazione urbana ordinaria e una extra-ordinaria quale quella rappresentata dal festival, la maggioranza dei bambini $(26 \%)$ ha risposto che Scarabò è anzitutto "divertimento", seguita a ruota $(23 \%)$ da "gioco".

Posto che le due espressioni virgolettate appena indicate, così come quelle che seguiranno, rispondono ad esigenze di categorizzazione e classificazione atte a razionalizzare e sintetizzare l'ovvia eterogeneità contenutistica dei pareri espressi, ciò che colpisce non è tanto il rimando all'aspetto gioioso e ludico, sebbene importante di suo e indicativo "forse" di un bisogno specifico oltre che di un'impressione prevalente, quanto l'associazione del divertimento e del gioco alla sfera interattiva-relazionale. Difatti, quasi la totalità delle risposte fornite "parla" al plurale.

Solo per fare qualche esempio (rif. "divertimento" con etichettatura anonima): B8, B10 (è divertirsi tutti insieme), B30 (ci si diverte con i compagni), B39bis (un progetto dove i bambini imparano divertendosi), B47bis (una cosa dove i bambini si divertono facendo laboratori). Altri esempi (rif. "gioco"): B26 (un posto dove i ragazzi giocano e si divertono), B32 (abbiamo fatto giochi diversi e imparato cose nuove), B48 e B49 (giochi da fare insieme).

In breve, rinviando ad un momento successivo le ponderazioni di carattere pedagogico, Scarabò è una festa (anch'esso termine da cui trapela il senso dell' "insieme": B13, B14bis, B22, B42) dove ci si diverte e si gioca insieme e, non ultimo, dove "si può" giocare e divertirsi insieme, ossia dove è consentito, diversamente da altri luoghi: B3 (un'attività dove si possono fare giochi), B11 (una cosa dove si può giocare, imparare e costruire), B12 (un posto dove si può giocare), B50 (è giocare in libertà), B14bis (una festa dove si possono imparare cose nuove).

A comprovare la rilevanza di quanto osservato tra le righe di queste prime due batterie di risposte, sembrerebbe poi giungere il terzo dato quantitativo, poiché il $13 \%$ degli intervistati ha fatto preciso riferimento alla dimensione dello "stare insieme". Ad esempio, senza riprendere opinioni a cavallo tra una categoria e un'altra: B13bis (mi è piaciuto perché sono stata con i miei amici), B19 (si sta insieme agli altri), B23 (tanti bambini e genitori che fanno lavoro e 
attività insieme), B36 (una grande attività per stare insieme e per andare per la piazza).

Per completare il quadro, seguono risposte che individuano in Scarabò un'attività educativa $(11 \%)$, una cosa molto bella $(9 \%)$, uno scarabocchio $(9 \%)$ - e in effetti Scarabò deriva giusto da scarabocchio -, un qualcosa che induce stupore $(3 \%)$, un luogo pieno di attività e persone $(2 \%)$, una città piena di colori $(2 \%)$, un progetto che coinvolge le scuole $(2 \%)$.

Queste prime risultanze e constatazioni parrebbero legarsi ed essere rafforzate, inoltre, dalle risposte maggioritarie (e non solo) offerte ai quesiti n. 3 "Che cosa hai imparato oggi?" e n. 5 "Vorresti che la città fosse sempre così? Perché?". Nel primo caso, 11 bambini su 53 (21\%), distaccando di molto gli altri responsi, hanno sostenuto di aver appreso la significatività di "stare/giocare insieme" con conoscenti, ma pure con sconosciuti: B10 (a stare con gli altri), B11 (a giocare con tutti), B13 (a giocare insieme ad altri bambini che non conoscevo), B14 (che ci si può divertire anche con persone che non conosciamo), B26 (che basta solo giocare con gli altri), B30 (a stare con gli altri bambini), B34 (a giocare con gli altri), B36 (che stare in piazza con gli amici è divertente e piacevole), B39 (a stare meglio con gli altri), B41 (stare insieme agli altri), $\mathrm{B} 48$ (che il bello è giocare insieme, non sempre vincere). Al secondo posto si posiziona un generico "ho imparato tante cose" $(13 \%)$ e al terzo posto, nuovamente, "che è bello divertirsi" ( $9 \%)$.

Nel secondo caso, 14 bambini su 53 (26\%) hanno affermato di volere la città sempre così perché è "divertente", seguita da un $17 \%$ che la vorrebbe così perché "è un modo per stare con altri": B9, B26 (sì, perché almeno si sta sempre in compagnia), B34 (sì, perché sto insieme agli altri), B35 (sì, così si incontrano nuove persone), B38 (sì, perché ci sono più bambini che mettono allegria), B47 (sì, perché possiamo fare cose belle ogni giorno), B48 (sì, perché è divertente stare insieme agli altri), B49 (sì, perché stiamo sempre insieme e c'è sempre pace e non guerra), B50 (sì, perché si fanno sempre nuove amicizie). Quindi, mentre al terzo posto (15\%) si colloca un "sì, perché è bella così/mi piace così", al quarto (10\%) abbiamo ancora a che vedere con la dimensione d'insieme: "sì, perché è piena di giochi da fare insieme".

A questo punto, se sembra ormai pacifico che lo stare insieme è un elemento prioritario, è interessante capire perché lo sia. E veniamo con ciò alla domanda n. 4 "Ti è piaciuto stare con gli altri? Perché?". I bambini e le bambine articolano su tre livelli le loro risposte:

1. il $34 \%$ asserisce che è piaciuto stare con altri perché ciò soddisfa un "bisogno di compagnia, di fare e stare con altri". Per esempio: B15 (sì, perché è bello stare in compagnia), B25, B26 (sì, perché insieme è meglio), B29 (sì, perché è più bello), B47 (sì, perché si lavorava insieme), B49 (sì, perché è un'emozione e una gioia stare insieme con gli altri); 
2. al $28 \%$ degli intervistati (seconda posizione) è piaciuto stare con altri perché "ho conosciuto nuove persone": B10 (sì, perché ho conosciuto nuovi amici), B13 (sì, perché ho conosciuto altri bambini, fatto nuove amicizie), B14, B16 (sì, perché ho fatto nuove amicizie), B17, B18, B23 (sì, perché ho conosciuto nuove persone), B32 (sì, perché ho conosciuto altri bambini e fatto amicizie diverse), B38 (sì, perché abbiamo costruito nuove amicizie), B50 (sì, perché ho avuto nuovi amici più grandi, non solo quelli della mia classe); 3. al $13 \%$ (terza posizione) è piaciuto stare con altri perché questi altri erano già compagni o amici noti: $\mathrm{B} 4, \mathrm{~B} 6$ (sì, perché sono venuta con due amiche), B12 (sì, perché ero con i miei compagni di scuola e mi sono divertito), B40 (sì, perché stavo con i miei compagni di classe e giocavo con loro), B42 (sì, perché c'era anche una mia amica), B44 (sì, perché mi diverto con amici), B53 (sì, perché sono miei amici).

Immancabile, infine, il riferimento al fatto che sia "più divertente" stare con altri ( $9 \%$, quarta posizione).

Divertimento, gioco, insieme. Queste, al momento, le tre parole più ricorrenti, le tre parole-chiave potremmo dire, con una predilezione netta per "insieme". Non resta che verificare se è stata avvalorata e dove si è concentrata la preferenza per questo insieme riguardo alle attività proposte. Alla domanda $\mathrm{n}$. 2 "Qual è l'attività che ti è piaciuta di più? Perché?", un'ampia maggioranza $(26 \%)$ ha risposto "caccia al tesoro". (Merita far presente che la caccia al tesoro, organizzata in vista di un altro progetto di ricerca internazionale da una collega del Dipartimento di Scienze della formazione, dei beni culturali e del turismo dell'Università degli Studi di Macerata, è stata strutturata per gruppi e dislocata in più luoghi del centro storico). Ecco alcune spiegazioni della propensione accordata: B8 (perché ho scoperto posti nuovi e sono stato con i miei amici), B9 (perché ho scoperto posti nuovi), B23 (perché è un lavoro di gruppo), B39 (perché ho notato particolari di Macerata che non conoscevo), B41 (perché divertente e ho fatto nuove amicizie), B47 (perché abbiamo corso e scoperto cose nuove), B49 (perché ha coinvolto tutti), B50 (perché abbiamo potuto accendere la mente). Ancora una volta un gioco, confermando l'apprezzamento per un' "attività d'insieme". A ciò si somma un elemento d'interesse ai fini del nostro discorso: la scoperta della città.

Per "dovere di cronaca", la seconda attività preferita è stata il "colorare/dipingere con le mani" (11\%), la terza il "giocare con la corda" ( $9 \%)$ a pari merito con il "gioco del calcio", la quarta "ballare" (7\%), la quinta "disegnare" (5\%).

Da ultimo, come vorrebbero i bambini le proprie città? Alla domanda n. 6, che intendeva sviscerare proprio questo aspetto, gli intervistati, con una maggioranza schiacciante (36\%), tenuto anche conto che il $23 \%$ non ha saputo/voluto fornire un riscontro, non hanno avuto dubbi nel rispondere che la vorrebbero come quella approntata da Scarabò, ossia colorata, piena di giochi da fare 
con altri e colma di persone in genere e di bambini. Mentre tutte le altre sono risposte singole e diversificate, il secondo responso quantitativo $(9 \%)$ ci informa che la vorrebbero con tanti giochi: $\mathrm{B} 6$ (con tanti giochi e attività nuove da imparare), B7 (con tanti giochi e bambini), B10 (con tanti giochi e musica per ballare), B26 (piena di giochi), B27 (con tanti giochi).

Come anticipato nell'incipit, a fronte dei risultati di sintesi avuti nell'esperienza di ricerca dell'edizione 2018 del festival, nell'edizione 2019 si è proceduto con l'approfondire il fattore "insieme", chiedendo al campione n. 2: "Oggi, a Scarabò, hai partecipato a delle attività con altri e hai giocato con altri, con bambini e adulti che conoscevi, ma anche con bambini e adulti che non conoscevi... Come è stato? Perché?". Ebbene, 24 bambini su 35 intervistati, hanno ribadito che la marcia in più, il bello di "Scarabò. Una città per educare", sta giustappunto nell'opportunità di stare con altri, specie se non conoscenti.

In proposito, come in precedenza, si riportano soltanto alcuni commenti, tra i più significativi: B3-19 (ho imparato cose nuove e soprattutto a stare bene con gli altri. Sarebbe bello vedere la città sempre cosi); B4-19 (mi è piaciuta tanto questa esperienza, perché stando insieme agli altri bambini ho potuto giocare con loro. Non mi preoccupava il fatto che non li conoscevo, anzi ho fatto finta di niente, come se avessi nuovi amici. Mi piacerebbe vedere sempre così la mia città); B7-19 (le attività mi sono piaciute, sono state belle, perché sono stata con altri bambini che non conoscevo); B10-19 (stare insieme ad altri bambini e adulti che conosco o no è stato bello perché almeno si fanno nuove amicizie e nuove attività. È bello vedere Macerata così, magari fosse sempre così anche nella mia città); B12-19 (ho conosciuto bambini nuovi con cui ho fatto amicizia e quindi mi sono divertito, perché giocare con qualcuno mi diverte di suo, più giocare con qualcuno di nuovo è ancora meglio); B20-19 (ho stretto nuove amicizie anche se conoscevo alcune persone. Quando giocavo con gli altri sentivo felicità ed è stato molto emozionante, è stato bellissimo veramente. Mi è molto piaciuto stare con gli altri perché ho fatto nuove amicizie); B21-19 (ero felice perché giocavamo tutti insieme, era piacevole. È più bello giocare con gli altri); B32-19 (ho imparato che, se anche non ci si conosce, si può giocare insieme, mi è piaciuto stare con gli altri, perché tutti ridevano ed è bello stare con il sorriso); B33-19 (eravamo in 18 a giocare e per questo mi sono divertita molto. Spesse volte mi piace anche stare sola a casa a giocare con i miei fratelli, però stare con altri compagni che neanche conosco bene è ancora più bello).

Per concludere, degli altri 11 bambini, 5 hanno partecipato ad attività solo con bambini o adulti che già conoscevano e non hanno stretto nuovi legami, 2 non hanno intrattenuto rapporti con alcuno, 1 non ha voluto giocare con alcuno e 3 non hanno rilasciato commenti attinenti alla domanda, hanno preferito parlare delle sensazioni dettate dal festival in generale. 
In dirittura d'arrivo, giungendo al campione n. 3 , talmente è varia la natura dei disegni raccolti (la cui pubblicazione, per ragioni d'ordine tecnico e di spazio, è rinviata alla monografia citata in apertura), unitamente ai relativi racconti, che offrire una rendicontazione di sintesi è impresa assai ardua. Perciò, mi limiterò a soffermarmi solo su alcuni contenuti, ricordando nuovamente la consegna: rappresentare se stessi mentre si gioca con altri in città, nella città che si vorrebbe.

B10D-19, 6 anni, sta disegnando la sua città, Montelupone, paese che dista pochi minuti di macchina da Macerata, e trasforma la torre realmente esistente in un castello. Tra il castello e la sua casa illustra una piazza, dove si situa con tanti altri bambini che giocano a palla e, nel farlo, spiega il tutto dicendo che nella sua città ci sono troppi edifici e che vorrebbe più spazi per giocare. B12D-19, 9 anni, di Montecosaro (altra cittadina poco distante da Macerata), vorrebbe una città simile ad un parco giochi, dove poter giocare con la sua amica, perché non ci sono tanti posti per giocare, pertanto disegna la sua città con uno scivolo, una piscina, palloncini colorati e molti giochi in una giornata di sole. B13D-19, 9 anni, ancora di Montecosaro, si raffigura insieme ad altri bambini mentre gioca con le bambole. Non c'è una città sullo sfondo, solo un cielo grigio, perché al momento del disegno stava piovendo - così dice - e sostiene che ha voluto disegnare gli altri senza città perché preferisce giocare con altri anziché in solitudine, perché giocando insieme impara nuove cose e si diverte. B15D-19, 4 anni, di Macerata, similmente a B12D-19, rappresenta la sua città come un parco-giochi, perché quando la mamma lo porta nel parcogiochi può giocare con altri bambini e divertirsi con loro. B17D-19, 8 anni, di Macerata, vera amante della lettura e vivace narratrice, non disegna alcun soggetto umano, bensì una scala fatta di libri che arriva fino al cielo, sorretta da una base di lettere dell'alfabeto, e un arcobaleno (per lei simbolo dei sogni aperti dalla lettura) vicino alla scala diretta verso le nuvole, perché con la lettura si può arrivare ovunque. Poi, una sola porta che introduce in città. Il motivo di questo disegno è presto detto: le piace tanto stare con gli altri, anche perché figlia unica, ma più di ogni altra cosa lei vorrebbe una città-biblioteca in cui possano entrare tutti, belli e brutti, con un solo ingresso, poiché, se anche le persone hanno origini diverse, entrano sempre dalla stessa porta e arrivano alle stesse vette a cui ti fa arrivare la lettura. B19D-19, 8 anni, di Macerata, si disegna insieme ad un'amica in una Macerata dove campeggia l'orologio della piazza centrale e tante bandierine colorate, come a Scarabò. Racconta che si è divertita molto a Scarabò, che ha conosciuto persone nuove e che, a volte, piange perché non ha nessuno con cui giocare, per questo, nel disegno, c'è lei con una sua amica.

Potrei proseguire con altri esempi, dedicati alla presenza di maggiore verde in città, o di case fatte di zucchero filato e cioccolato, o di festoni colorati, ma 
è sufficiente la carrellata esposta. In generale, altresì, giova assicurare che, a corredo di quasi tutti i disegni, eccetto uno soltanto realizzato da un "amante della solitudine", i bambini hanno sempre tenuto a precisare che giocare o fare attività con altri è di gran lunga la cosa migliore. Infine, vale la pena sottolineare di nuovo che ogni risposta e narrazione riportata fin qui rispecchia fedelmente le parole usate dai protagonisti, senza alcuna alterazione.

\section{Premessa alle riflessioni pedagogiche: educazione permanente e città educante}

A circa venti anni dall'avvio del dibattito pubblico sull'educazione permanente, Mencarelli (1986), in dialogo con Richmond e Suchodolski, e indirettamente con Dewey, più volte richiamato nel corpo del testo, riflette sul rapporto tra tale idea-guida e la democrazia, ponendo in luce i vari ostacoli che si impongono all'affermazione del «valore» educazione permanente, anche quale volano di prosperità democratica. Tra questi, citando proprio il Dewey (1946) di Liberalismo e azione sociale, v'è la crescente riduzione dell'uomo ad homo oeconomicus e il progressivo confinamento della «creatività culturale» nella sfera economica, lasciando che l'impegno educativo e specialmente autoeducativo, cuore pulsante della stessa educazione permanente, si risolva in una mera funzionalizzazione al servizio dell'agire economico. L'educazione permanente, al contrario, ribadisce Mencarelli, non volendo peraltro espungere dalla scena dell'orizzonte realizzativo umano la dimensione economica, intende trascenderne la pretesa di protagonismo assoluto per estendere il «principio dell'essere» su quello dell' «avere» e attualizzare un «umanesimo dell'autenticità». L'educazione permanente è l'anima di uno spirito "demo-cratico" in quanto, fondamentalmente, "cultura emergente», ossia risposta dinamica ad istanze culturali altrettanto dinamiche che si fanno latrici di specifiche esigenze di «emancipazione», di «espressione» e di «convivenza gratificante». Di più ancora, l'educazione permanente disegna e promuove democraticamente il «diritto ad essere», cioè a «vivere con pienezza umana», invocando con ciò la libertà autorealizzativa delle persone (da condizionamenti di marca tecnico-economica su tutti) e la libertà della «vocazione culturale» emergente, la quale, per esprimere al meglio i bisogni che si propone di veicolare e soddisfare ai fini di una crescita in umanità, ha da essere supportata da un onere educativo partecipato, da un' animazione socio-culturale a muovere dal basso, dai vivi dinamismi di una comunità che voglia dirsi e farsi educante. Del resto e per inciso, tornando a Dewey (1949) - il quale invero non ha mai formulato una teoria sull'educazione permanente, ma ha sempre perorato la causa di una «società genuinamente educativa», perché «"'l'educazione desiderata non può avvenire entro le quattro mura di una scuola separata dalla vita"» (Richmond, 1986, pp. 
47-48) -, l'attuazione delle potenzialità di ciascuno concepita come senso ultimo della democrazia è in stretto rapporto con un'efficienza sociale determinata dalla possibilità di coltivare insieme valori dal basso rigenerando la cultura (Spadafora, 2011).

Perché questo riferimento a Mencarelli e quanto ne segue? Per quattro ordini di motivi. Primo, perché il rimando al paradigma dell'educazione permanente (con l'annessa comunità/società educante) e al suo esplicito anelito democratico, vividamente rappresentato dalla sua propensione a nutrire il pensiero critico-creativo delle persone, puntellare la convivialità delle differenze e contrastare ogni forma di alienazione del potenziale umano per mezzo dell'educazione totale di tutti (Lorenzetto, 1976), anziché puntare primariamente a mete economiche, è il rimando al paradigma entro cui si origina il concetto di città educante a cui si ispira apertamente il nostro Scarabò in piccolo e a cui mi sono rivolto per gli studi dedicati al progetto Trans-Urban. Studi che, peraltro, rivendicano il distanziamento dall'idea più recente di learning city (città che apprende) (Longworth e Osborne, 2010a; Longworth e Osborne, 2010b; Jordan, Longworth, e Osborne, 2013). Questa affonda le proprie radici nel paradigma del lifelong learning e palesa inclinazioni economicamente funzionalistiche, vincolate alla competitivà "glocale", all'incremento competenziale per il mercato, ecc. - così come riconosciuto pure da uno dei più autorevoli studiosi della medesima learning city (Longworth, 2007) -, sebbene si stia tentando in ultima battuta il recupero di una visione più etica e umanistica del learning (Osborne, Kearns e Yang, 2013) propugnando la città quale luogo privilegiato di incontro tra sollecitudini educative e sviluppo democratico (UNESCO, 2013). L'idea di learning city, tuttavia, pur prevalente oggigiorno, non ha affossato quella di educating city: dal report Recurrent Education dell'OECD (1973), che sollecitava la valorizzazione delle città educanti, fino al primo congresso internazionale delle città educanti di Barcellona (1990), che ne ha recepito compiutamente la raccomandazione redigendo la Charter of Educating Cities, la successiva International Association of Educating Cities, sorta nel 1994 a Bologna, conta ad oggi 499 città nel mondo (cfr. www.edcities.org./en/).

Secondo, perché al centro di quella prospettiva di educazione permanente che dà corpo al progetto "eu-topico" della città educante (Borello, 1989) c'è per l'appunto un diritto ad essere che sintetizza in sé tutta una serie di diritti personali e sociali il cui appagamento è finalizzato all'umanizzazione dell'uomo e dell'ambiente in cui abita, ivi incluso il diritto al valore d'uso della città e non di scambio, come direbbe Lefebvre (1970).

Terzo, perché il suddetto appagamento è conseguibile, in primis, attraverso l'impegnata animazione dal basso di una comunità che, con creatività culturale, si prefigge lo scopo di accogliere e far emergere premure educative e appren- 
ditive dando loro voce, concorrendo finanche al ripensamento della cultura, degli orientamenti e dei valori educativi in essere. E questo è ancora il caso di Scarabò.

Quarto, proprio per l'allargamento del raggio d'azione educativa e formativa all'intera comunità, al di là della sfera d'influenza scolastica, ambendo in questo senso ad un'integrazione tra diversi livelli formali e informali che, invero, costituisce, non solo il proposito reticolare delineato da Scarabò in ordine ad un affiancamento delle attività scolastiche (Trilla Bernet, 2005), ma pure il principio fondante una città educante (Frabboni, 1990; 1991).

Tutto ciò doverosamente premesso, si può osservare come la sintesi delle considerazioni prodotte sia in stretto rapporto con i risultati dianzi riportati. Il diritto ad essere, concepito come diritto a vivere nella pienezza umana secondo la logica etico-morale dell'educazione permanente, è anche, come abbozzato, il diritto ad "usare" una città per la propria crescita e il proprio sviluppo umani in luogo della secca coincidenza con il valore economico, quindi commerciale e consumistico, dei suoi spazi, accostando esigenze propriamente educative ad esigenze economiche. Difatti, quando si pensa ad una città educante (Fernando e Morell, 1990; IAEC, 2008), per certi versi sin dal suo ritratto aurorale (Ortega Esteban, 1990; Trilla Bernet, 2005; Stenger, 2019), si pensa ad un contesto capace di favorire l'educazione permanente dei cittadini e di trarre diversi vantaggi da essa e da una diffusa disponibilità all'apprendimento, tanto sulla sponda del progresso democratico, del benessere personale e comunitario, della cittadinanza attiva, della coesione sociale, dell'integrazione/inclusione delle risorse, ecc., quanto su quella, non esclusiva, dei benefici economici. Alla base di siffatta facilitazione e della sua occorrenza, c'è il fatto che le persone hanno urgenze apprenditive e potenzialità educative che dovrebbero poter soddisfare e concretare nei luoghi in cui vivono (Piazza, 2013), grazie all'impegno educante della comunità tutta, il suo contributo in vista dell'implementazione dei processi educativi/formativi e, nondimeno, la disponibilità di spazi in sintonia con le esigenze educative di ogni fascia d'età, a partire dai più piccoli (Gennari, 1989).

In altri termini, diversamente dall'attenzione preminente riservata agli output economici da parte della learning city, una città educante è sia la città in grado di "liberare" gli itinerari educativi dei propri abitanti in un'ottica "permanente", coinvolgendo e integrando differenti attori e istituzioni, così come ampliando e arricchendo la propria proposta educativa, formativa, informativa e culturale, e sia la città che riflette pedagogicamente sui propri spazi.

Riguardo a quest'ultimo punto, la "cultura emergente" che sembrerebbe discendere dalle interviste effettuate, a fronte delle dinamiche educative attivate, pare orientare limpidamente verso la domanda di convivialità, o ieri come oggi di "convivenza gratificante" per il proprio poter/dover essere. Ma esistono nella 
città di Macerata, come in altre città, luoghi conviviali di incontro "gratuito"? E ne esistono specialmente per i bambini e le bambine, per i loro bisogni ludici e relazionali?

\section{Riflessioni pedagogiche sui risultati}

\subsection{Criticità urbane e bisogno di convivialità}

Ripiegando sul predominio delle ragioni economiche lamentato da Mencarelli, e di certo acuito nella contemporaneità, le città di oggi sembrano sempre più scarno territorio economico e crocevia di funzioni produttive imprenditorialmente decontestualizzato, subordinato alle logiche commerciali (Amadini, 2012). Se appare arduo addentrarsi in questa sede all'interno delle complesse ponderazioni espresse intorno al rapporto tra capitalismo e produzione dello spazio (Harvey, 2019), è sicuramente più agevole e non di certo anacronistico comprendere con Tizzi (1989) che, attualmente, nelle città, l'investimento maggiore di senso concerne i negozi, le strade/aree commerciali, i simboli e i luoghi del consumo.

Sebbene Macerata sia una piccola città di provincia, che conta solo 42.000 abitanti, non è affatto esentata da questa rappresentazione. Come non lo è dalla difficile praticabilità e percorribilità urbana. Essa, come altre città, è prevalentemente un autodromo fatto per l'uomo motorizzato, come sottolinea Binanti (2007), con tutto quello che ne consegue circa l'espropriazione infantile della città, la pericolosità del suo attraversamento sul piano della sicurezza e anche dell'inquinamento ambientale, la paura genitoriale che possa accadere qualcosa di infausto e la corrispondente limitazione di movimento imposta ai figli, accompagnati ovunque. Paura e inibizione del movimento, come ricorda uno studio curato da Hillmann (1993), che, poi, finiscono per minare il senso di autonomia dei piccoli ricadendo per di più sul senso di autostima, e finiscono per ingenerare a loro volta, nei bambini, paura per l'ambiente circostante.

A tutto questo si aggiunge, ulteriormente, la mancanza di oasi per i bambini (Gennari, 1995) che, da un lato, non siano spazi-gioco strutturati, ossia lineari, per dirla con Sennett (1992), inadeguati a lasciare campo libero alla fantasia, all'interpretazione, alla scoperta, all'avventura, alla narrazione e, dall'altro lato, che non siano costellati di divieti (restringenti il gioco, di calpestio dell'erba, ecc.) (Bertolini, 1989); l'individualizzazione e la privatizzazione degli spazi, la quale porta a separare nettamente interno ed esterno e privato-pubblico, privando i bambini, e non solo, di zone di interazione e svago "al confine" (Iori, 2003), così come di occasioni di esperienza ed esplorazione ambientale extra- 
privato (Bertolini, 1989; Bertolini e Cardarello, 1989; Amadini, 2012); l'uniformità estetica e la standardizzazione architettonica delle nuove impostazioni abitative ai margini del vecchio centro storico (Gennari, 1995); la proliferazione di nuovi edifici abitativi e commerciali a discapito di aree di socializzazione; e, tornando alle criticità relative al movimento e pure alla privatizzazione degli spazi, ma andando oltre il tema della sola "percorribilità" urbana, il problema di una "scoperta" sicura, autonoma e libera della città, con ancora riverberi sull'immagine di sé e del contesto di vita (Vercesi, 2008).

A queste problematiche, affrontate da vari studiosi e d'interesse comune per Macerata, se ne può annettere un'altra. Macerata è una città essenzialmente universitaria, i cui residenti lavorano per la maggior parte fuori dal centro, in periferia o nelle varie frazioni, ovviamente ad eccezione di chi lavora per l'accademia, non di rado residente all'esterno di Macerata. Per questo, nel tempo, si è gradualmente svuotata durante il giorno, rimanendo perlopiù animata nelle sere del fine settimana, durante il mercato settimanale del mercoledì mattina e nei mercoledì e giovedì sera dagli studenti universitari. Nei giorni restanti, la città è poco frequentata, in particolar modo il centro storico. Inoltre, comunemente ad altre città, il suo cuore abitativo si è ormai allargato alle periferie, comportando una dilatazione/articolazione dello spazio vitale cittadino e un diradamento della presenza nel centro stesso, il quale, è bene farlo presente, è solo parzialmente pedonale.

Ora, completato il quadro, di fronte agli elementi passati in rassegna i bambini intervistati sembrerebbero avere le idee chiare ed esprimere bisogni altrettanto chiari, che ho prima riassunto con il bisogno di convivialità o di convivenza gratificante. In effetti, sulla scorta delle risposte date in forma maggioritaria e al cospetto di un'analisi di primo livello dei risultati, potremmo sostenere, riepilogando, che essi avvertano l'esigenza di divertirsi e giocare insieme, di un posto come Scarabò dove potersi divertire, giocare e imparare assieme, di apprendere a stare, giocare e fare con gli altri, di una città come quella allestita da Scarabò perché divertente e perché li fa interagire con altri. In più, parrebbero evidenziare il bisogno di compagnia, di evitare la solitudine delle mura domestiche, di conoscere persone nuove, o di passare semplicemente dei momenti con gli amici. Infine, vorrebbero una città da scoprire, con più aree per giocare insieme, colorata, piena di persone e di bambini.

Una città a misura di bambini e bambine. Questo potrebbe essere lo slogan capace di sintetizzare il tutto. Accompagnato da una parola-chiave: interdipendenza. Il valore principale che sembra emergere dall'indagine e che i bambini ci sollecitano a ricomprendere è proprio quello dell'interdipendenza positiva, perché, come ci insegnano, "insieme è meglio". 


\subsection{Recuperare la funzione relazionale della città}

E, allora, che cosa fare? Intanto, riappropriarci di una città a misura di bambini e bambine, a partire dal centro storico, come nell'intenzione di Scarabò.

Se i bambini ci esortano a riflettere sulla rilevanza dell'interdipendenza, stimolati da un evento come Scarabò, Scarabò, a sua volta, pur sempre nel suo piccolo, ci insegna, non solo che si può integrare l'attività svolta dalle scuole (e ugualmente dall'università e da altri enti) dando realisticamente corpo all'idea di una città educante, in grado di ampliare le esperienze formative per l'infanzia, l'adolescenza e pure l'adultità, ma anche che, attraverso la mobilitazione dal basso, volontaristica e priva di lucro, partecipata e impegnata, di una comunità fatta di educatori e di gente che non svolge professioni educative, semplici cittadini al servizio della cittadinanza e della sua crescita, è possibile riappropriarsi di una città nel senso primigenio del suo ruolo. In proposito, Bertolini (1989, p. 50), spiega bene che cosa si intenda con riappropriarsi, parlando di «riguadagnare il possesso della città»: «se vogliamo che la città diventi uno spazio pedagogico [perché è certo che sia un oggetto educativo ma non che sia uno spazio pedagogico] occorre far sì che il cittadino non sia sottomesso alla struttura incombente della città che lo passivizza e lo condiziona in senso negativo. Il vero scopo da perseguire è fargli riguadagnare il possesso della città», ridestando «relazioni interpersonali autentiche».

In fondo, il primo, grande merito di Scarabò è giusto quello di procedere in direzione di siffatto riguadagno, ponendo al centro di tutto il suo progetto la riaccensione di relazioni interpersonali gratuite, non funzionali o strumentali, agendo con e sulla creatività culturale rammentata. Per vari motivi, oggi, le persone hanno smarrito il senso di comunità: assuefazione all'evoluzione dei tratti caratteristici dell'homo oeconomicus, adesione ad una cultura individualistica e malsanamente performativa e competitiva, ripiegamento narcisistico su di sé, chiusura nella casa-rifugio, paura della diversità in un mondo globalizzato, ecc. (Bauman, 2003a, 2003b; Bazzicalupo, 2013; Dardot e Laval, 2013; Lordon, 2015; Chicchi e Simone, 2017). E a farne le spese relazionali sono i grandi, quanto inevitabilmente i piccoli. Eppure, il bisogno di comunità continua ad ardere sotto la cenere di un'autosufficienza baldanzosa ma fragile (Perucca, 2007). E quando opportunamente solleticato, esplode in tutta la sua forza, cercando soddisfazione.

È facilmente intuibile che i bambini, nelle loro risposte, abbiano esaltato la significatività dello "stare insieme" perché hanno avuto l'occasione di sperimentarlo in profondità, passando due intere giornate a contatto con altri, facendo cose facili e difficili insieme (Sennett, 2012), giocando insieme, e ne siano stati condizionati positivamente, tornando all'espressione usata da Bertolini. Al di fuori della scuola o dello sport di squadra, per chi lo pratica, i bambini 
di oggi, come abbiamo potuto notare, non hanno molte opportunità di godere della presenza dell'altro nelle loro città, permettendo di riconoscersi vicendevolmente, di strutturarsi identitariamente nel confronto, di intrattenere insomma relazioni educative significative. E, allora, ecco perché vorrebbero una città sempre così: perché dà loro modo di vivere una condizione che mai troppo spesso riescono a vivere. D'altra parte, anche gli adulti - anticipo un dato inerente ad un altro "pezzo" della ricerca effettuata - vorrebbero che la città fosse sempre così proprio per il medesimo motivo. E vorrebbero che Scarabò fosse reiterato più volte durante l'anno, o più volte al mese, o tutte le settimane. Quel bisogno di comunità di cui sopra è affiorato nell'incontro, nella "con-versazione", nell'interazione ludica, nella cooperazione, con altri adulti, con bambini e con ragazzi, e ha lasciato un segno, "in-segnando" pure a loro che insieme è meglio e che il centro della città può essere rivisto con occhi diversi, con uno sguardo relazionale.

Ora, ancorché sia volontà dell'ideatrice del festival dare sistematicità temporale all'iniziativa, e si sia già adoperata per questo organizzando altri Scarabò nei quartieri tra il 2019 e l'anno in corso (denominati Scarab-out), non possiamo pensare che ciò risolva in toto le varie criticità. Però, possiamo lecitamente ipotizzare che eventi di questo genere possano nutrire il desiderio di recuperare la funzione relazionale della città.

\subsection{Spazi a misura di bambini e bambine}

Tuttavia, a siffatta funzione non è dato di manifestarsi se non esistono luoghi idonei. A Scarabò è concesso di usufruire di un centro storico liberato da automobili, con le vie, le piazze, le corti interne interamente disponibili e con la sospensione dei confini tra pubblico e privato. Ma nella vita di tutti i giorni non è così. Pertanto, il secondo merito di Scarabò è quello di ospitare, curare e rilanciare - anche con questo articolo - il bisogno di dimorare spazi «'attivamente inutili'» e sicuri per potersi esercitare nel «tirocinio ludico» della città (Amadini, 2012, pp. 26 e 28), intessendo con il «gioco autentico» (Perucca, 2007 , p. 47) trame relazionali congeniali alla costruzione dell'essere e del "conessere".

Per spazi attivamente inutili si intendono spazi non circoscritti ad un rigido e circostanziato utilizzo, bensì aperti a nuovi e sempre diversi approcci, abitabili dai bambini e dalla loro varia attività sul mondo, anzitutto ludica. I bambini hanno il diritto di non ridursi a meri consumatori di luoghi già codificati per il loro uso, ma di agire e interpretare assieme, creativamente, la realtà circostante e i suoi materiali, al fine di "significarsi" personalmente e, ad un tempo, conferire "significati mediati" all'esperienza di sé, dell'altro da sé e del mondo stesso (Perucca, 2007). Così, avvalendosi di spazi “dischiusi”, il tirocinio ludico della 
città, prima che a semplice modalità di socializzazione, assurge a modo etico «dell'essere-con-l'altro» (Amadini, 2012, p. 26), perché nel vivere ludicamente la città tramite il gioco condiviso, libero, autentico - quello che ricrea e rigenera il rapporto con la suddetta realtà, le cose, le situazioni, i soggetti -, «il bambino incontra gli altri ed impara a concordare con loro il gioco serio, complesso e difficile della vita» (Perucca, 2007, p. 47). Ovverosia, agli effetti di un'adultità matura, accogliente e "respons-abile", impara a cogliere il valore dell'alterità, della differenza e della conciliazione dei punti di vista per la propria crescita, per l'attribuzione di senso al contesto di appartenenza, per il rafforzamento del senso medesimo di appartenenza e di partecipazione, per l'arricchimento della cultura comune, per l'edificazione di una cittadinanza democratica.

Viceversa, predisporre spazi-gioco "chiusi", pur nel verde come esistono a Macerata e altrove, non serve adeguatamente la causa di una relazionalità educativa autentica e significativa. Possono sì appagare una domanda ludica e di compagnia, ma essendo perlopiù strutturati e lineari, come anzidetto con Sennett, ed essendo prevalentemente votati ad una "pausa di divertimento" isolata che elude il confronto con il mondo (Perucca, 2007), non possono parimenti dar adito ad una "con-generazione" interpretativa e di senso della realtà vera e del proprio posto in essa. È la città, a muovere dal suo centro nevralgico e dalle vie, piazze, vicoli, porticati, ecc. in cui si articola, che deve in primo luogo prestarsi ad essere attivamente inutile e "manipolabile" ludicamente dai suoi cittadini più giovani, spalancando loro la strada verso la libertà di essere e "conessere" nella concretezza della sua vita quotidiana, e non nell' astrattezza di luoghi omologati.

Il discorso fin qui affrontato ha indubbiamente a che fare con l'accessibilità sicura alla città e con la revisione della mobilità in favore dell'infanzia (Tonucci, 1996); con la pedonalizzazione degli spazi del centro e non solo; con lo sfilacciamento dei perimetri di privatizzazione; e, non ultimo e in connessione con il resto, con le richieste già evidenziate di una città esplorabile (Trisciuzzi, Nuti e Viaggi, 1993), andando a rimuovere impedimenti fisici e divieti che ostino la soddisfazione di un bisogno di scoperta, prefigurazione fantastica, avventura e conoscenza, oltre che ludico, segnatamente importante per l'età preponderante dei bambini intervistati, come rimarca ancora Amadini (2012) aderendo al pensiero montessoriano. E tutto questo, a sua volta, rinvia al tema della progettazione della città e del concorso pedagogico ad essa (IAEC, 2009; Million, Heinrich e Coelen, 2017).

\subsection{Ripensare progettualmente la città}

In una città pensata eminentemente per l'adulto, dove la presenza ineludibile degli altri per il gioco autentico del convivere è distratta da pericoli, vincoli e 
attraversamenti funzionali o commerciali, dove l'homo faber impera a svantaggio dell'homo ludens, dove non v'è pertugio per il «robinsonismo» e la "ricreazione" dei piccoli, il contributo pedagogico al ripensamento progettuale del tessuto urbano è essenziale e non può prescindere dall'avvaloramento della dimensione ludica e generalmente infantile (Scurati e Lombardi, 1982, p. 213).

A dedicarsi nello specifico a questo tema è stato Gennari $(1989,1995)$, il quale afferma che il presupposto affinché una città possa dirsi educante risiede nella sua stessa struttura. Ė la struttura ad indicare se vi sono o meno condizioni di educabilità o virtualità educative. Perciò la pedagogia ha da incunearsi tra l'urbanistica e l'architettura per cercare di assicurare che vengano tenute in debito conto le necessità educative di ciascuno in relazione alla fruibilità degli spazi, non solo scolastici, ma anche sociali. Ciò non vuol dire che occorra stravolgere il volto di una città, tanto più se il pensiero va a città che hanno una storia secolare e che, per molti versi, sono "intoccabili". Piuttosto, al di là del ragionamento intorno a nuove costruzioni, vuol dire che si può (e si dovrebbe) intervenire per apportare modifiche d'impronta educativa, in modo da consegnare significato educativo ai segni che costituiscono il "testo urbano". Per esempio, rivolgendo lo sguardo alla succitata inutilità attiva degli spazi urbani, un certo uso di colori, luci e sonorità potrebbe agevolare la ricomprensione di questo "testo" in chiave infantile e più ampiamente di usufrutto socio-relazionale, senza snaturarlo dalle fondamenta. «Una città attenta alla qualità della vita è $[. .$.$] anche uno spazio urbano disponibile ad essere riconosciuto come spazio$ educativo. E una città che voglia essere educante non può trascurare di modificare il proprio tessuto urbano [...] in funzione anzitutto dell'infanzia» (Gennari, 1989, p. 149), garantendo, oltre la sicurezza, opportunità di stimolo conoscitivo, di incontro, ludiche e di scoperta.

Questa sottolineatura «richiama il punto d'osservazione pedagogico, non esitando a cogliere nella formazione dell'uomo la misura decisiva della [modificazione] della città. Quando i linguaggi della città parlano i lessici dell'umano, l'oggetto e il soggetto si incontrano in una prospettiva di mutua convergenza», ribadendo che una città educante è, in primis, «l'esito di un progetto per l'uomo». «Una città vissuta [...] nella scoperta dei suoi testi storici, artistici, culturali, naturali; una città abitata in ogni suo luogo e in ogni suo tempo senza esitazione, rimpianto o paura; una città che accolga nelle proprie piazze le presenze e i significati profondi dell'intersoggettività [...]; una città, infine, che sia un mondo dell' 'esperienza' aperto [...]: ebbene una consimile città risolve l'opposizione tra urbanesimo e umanesimo a favore di quest'ultimo. Il suo centro resta l'uomo, mentre il centro del suo centro evoca il rispetto dell'essere» (Gennari, 1995, pp. 19 e 21).

Ciò nondimeno, l'apporto pedagogico al ripensamento progettuale e al cambiamento delle trame urbane si sostanzia pure nel sollecitare la presa in carico 
delle istanze dei diretti interessati a livello di scelte politiche, quindi urbanistiche e architettoniche (Million, Heinrich e Coelen, 2017). I risultati emersi dalla ricerca, in effetti, potrebbero assumere un diverso peso, se idoneamente ascoltati e armonizzati all'interno di un disegno co-progettuale. In quest'ottica, puntare su una progettazione partecipata con i bambini consentirebbe, non soltanto di approfondirne i bisogni e di dar sbocco ai loro desideri di socialità apprenditiva e ludica e di maggiore interazione conoscitiva con l'ambiente, ma anche, con appropriati mezzi di mediazione, di favorire una vera e propria processualità formativa, in virtù dell'attivazione di scambi simbolici e relazionali, di un pensiero critico-creativo, di energie emotive, di autodeterminazione, insomma di tutta una serie di potenzialità che verrebbero incitate a manifestarsi (Amadini, 2003).

Con il rimando alla progettazione partecipata, si richiama l'importanza di integrare i punti di vista per trasformare la città, di condividere responsabilità che incrementano il suddetto senso di appartenenza, di co-costruire un orizzonte di senso comune entro una prospettiva democratica di scelta e decisione volta al bene comune, non subordinando l'angolazione squisitamente educativa a quella propriamente tecnica e riconoscendo ancora l'interdipendenza come valore da onorare e promuovere.

\subsection{Due proposte per una città (anche) dei bambini}

In dirittura d'arrivo, infine, due proposte di facile attuazione per venire incontro ai bisogni degli intervistati, fermo restando che offrire continuità a Scarabò, nelle sue molteplici forme, e intensificarne la presenza temporale si confermerebbe quale soluzione ottimale per molte delle criticità riscontrate, incidendo tanto sul versante dei risvolti ludico-apprenditivo-educativi, quanto su quello dell'animazione socio-culturale e sul rafforzamento dello spirito comunitario al servizio della crescita umana della città.

La prima proposta riguarda l'impatto estetico del centro storico. Nel corso di Scarabò, il centro è adornato di festoni colorati che sovrastano le sue arterie principali e laterali, di insegne colorate poste sopra ai gazebo o ai portoni d'ingresso degli edifici o alle volte delle corti che ospitano i laboratori, concorrendo ad infondere un senso di festa ben percepito dai bambini e diffusamente proiettato nello loro "immagini" di una città ideale. Difatti, la vorrebbero sempre piena di colori. Ecco, perché non lasciare che almeno i festoni, se non insegne ad hoc, permangano come addobbo duraturo, durante tutto l'anno? Costituirebbero una spesa lieve, appagherebbero un desiderio sostenibile e restituirebbero idea e segno percepibile di una città inclusiva nel concreto, capace di porsi in ascolto di tutti, compresi quelli che solitamente hanno meno voce in capitolo. 
Se la prima proposta, ancorché piccola cosa ma contemporaneamente grande in termini di accoglienza dell'infanzia nella "città adulta", elude il tema centrale affiorato dall'analisi dei risultati, la seconda le è pienamente attigua. Perché, quanto meno nei fine settimana, non rendere integralmente pedonale il centro storico (con buona pace dei residenti e delle loro occorrenze di mobilità automobilistica interna) e costellare le diverse piazze e piazzette di box visibili e accessibili a tutti, contenenti materiale utile per giocare insieme, bambini e adulti? Si potrebbero usare le piazze come fogli da disegnare e colorare, oppure per giocare a "ruba bandiera", o per il tiro alla fune, ecc. E il centro potrebbe così tornare ad essere pure luogo extra-commerciale, di incontro e di relazioni, educativo, rinnovando costantemente quell'invito a "stare insieme" caro ai più piccoli come ai grandi.

\section{Conclusioni}

La ricerca effettuata non svela qualcosa di sorprendente e inaudito. I profili e i volti delle nostre città sono sotto gli occhi di tutti. Così come è consapevolezza comune che i bambini di oggi godano sempre meno di spazi di interazione, peraltro progressivamente surrogati e surrogabili dai social media.

La ricerca, semmai, grazie all'evento Scarabò, ha il pregio di porre in luce una situazione contestualmente specifica, sottolineando esigenze locali che, altresì, trovano per certi versi riscontro a livello nazionale, se è vero che nella multidimensionalità della povertà educativa - come descritta da Save the Children (2018) a seguito della consultazione di centinaia di minori in tutto il Paese, ispirandosi alla Convenzione ONU sui Diritti dell'Infanzia e dell'Adolescenza e prendendo spunto dal capability approach di Sen e Nussbaum - rientra la privazione legata all' «apprendere per vivere assieme», o «capacità di relazione interpersonale e sociale». In questo senso, nell'ultima pubblicazione, centrata sul rapporto tra povertà educativa e resilienza in Italia, si sostiene che «le relazioni sociali ed emotive intessute $[\ldots]$ nella comunità educante, le opportunità formative presenti nell'ambiente che circonda il bambino possono rappresentare $[. .$.$] dei fattori di protezione o al contrario di rischio». E ancora: «l'am-$ biente che circonda il minore [...] è essenziale per l'acquisizione di quelle abilità definite 'non-cognitive', fondamentali per apprendere e vivere nel mondo di oggi, complesso, [...], quali la motivazione, la fiducia in se stessi, la perseveranza, le aspirazioni. La comunità 'educante' influenza fortemente la capacità dei bambini di superare le difficoltà» (Save the Children, 2018, pp. 15-16).

Il rinvio, proficuo, alla comunità educante, consente di affermare che il secondo pregio attribuibile alla ricerca svolta è quello di aver contribuito ad aggiornare lo stato dell'arte degli studi sulla città educante. Come si avrà avuto 
modo di constatare, gran parte della letteratura esistente in materia è abbastanza datata e si concentra in un periodo preciso, connesso con il fermento educativo che avrebbe portato al summenzionato congresso di Barcellona e che ne è evidentemente seguito. Come già osservato, con il graduale sovrapporsi del paradigma del lifelong learning su quello dell'educazione permanente, anche le riflessioni e le indagini empiriche sulla città educante si sono diradate, preferendo curvare l'attenzione sulla più recente città che apprende. Nonostante questo dato di fatto, il filone originario non è andato perduto e in questa sede se ne è dato conto, sebbene sia pacifico che l'oggetto non sia particolarmente frequentato nell'attualità. Ad onor del vero, in ambito nazionale, non lo è nemmeno quello della learning city, che conta maggiori estimatori in ambito internazionale (Yang, 2012; Kearns, 2015). Tuttavia, per l'idea guida rappresentata dall'educazione permanente, per il suo afflato etico-morale, per il suo rispetto dell'essere e per la missione autenticamente democratica che incarna e promuove, ponendosi come fine l'attuazione delle potenzialità di ciascuno prima che obiettivi economici, ritengo che la tematica della città/comunità educante che si collega ad essa abbia da essere coltivata ulteriormente e con maggiore impegno. Questo articolo e ciò che ne seguirà, continuando a pubblicizzare altri risultati in altre sedi, è, dunque, anche un invito rivolto ai colleghi pedagogisti, affinché riprendano a cimentarsi in un terreno di coltura educativa assai rilevante da molteplici punti di vista, interessando di fatto le più giovani generazioni e il loro sviluppo, l'adultità, l'invecchiamento attivo, le dinamiche relazionali, quelle familiari e sociali, le problematiche ambientali, l'integrazione e l'inclusione sociale, ecc.

Il terzo pregio, proseguendo, è senza dubbio da individuarsi nell'aver amplificato la voce dei bambini, sottoponendo le loro risposte e le loro istanze, non solo all'attenzione del panorama scientifico, a muovere dagli urbanisti e architetti che partecipano numerosi al progetto Trans-Urban, ma pure ai decisori politici, auspicando che si mettano in ascolto e che possano trovare spunti per una modificazione urbana e per una dedizione comunitaria allargata in ciò che è stato illustrato. Non si può cavalcare l'onda odierna della sostenibilità urbana e farne spesso una bandiera politica senza partire dalle esigenze dei più piccoli, senza recuperare quel gioco del "con-essere" che sta alla base della formazione umana dell'uomo e di cittadini responsabili e coesi, animati da una volontà di appartenenza ed impegno comune ("cum-munus", comunità) tesi al bene di tutti e ciascuno. La meta dell'inclusione e della città socialmente integrativa, per tornare al soggetto di Trans-Urban, passa inevitabilmente per una città accogliente, in grado di ospitare, rendere disponibili e alimentare intrecci relazionali che permettano quella reciprocità di riconoscimento che si fa dono di cura per se stessi e per gli altri, per un noi autoeducante. La sostenibilità 
urbana è, sì, sostanziata da criticità da risolvere in ordine alla viabilità, alla mobilità, all'inquinamento, alla coesistenza tra fattori produttivi e ambientali, alla "buona" edificazione, all'estetica, all'armonia paesaggistica, all'adeguamento tecnologico, ecc. Però, prima di tutto, ha a che vedere con il fulcro attorno cui ruotano tutte queste questioni, ossia il benessere delle persone che vivono la città. D'altra parte, il "ben-essere" non può dirsi autentico e costitutivo di un'arricchente progettualità esistenziale se la persona non ha occasioni di poter crescere in umanità attraverso l'esperienza della significatività auto e co-realizzativa ritracciabile nell'incontro con l'alterità, dunque se non ha opportunità di sperimentare relazioni gratuite, relazioni di dono educativo per l'appunto. Siffatto tirocinio dell'incontro e della differenza dovrebbe essere puntellato in famiglia, certamente, a scuola, ma nondimeno dovrebbe essere garantito nello spazio di vita cittadino, concedendogli una morfologia formativa e la praticabilità ricorrente di eventi formativi comunitari e comunitariamente implementati in rete. $\grave{E}$ anche in questo spazio che avviene il farsi persona nella pienezza ontologica e valoriale delle proprie qualità. Qui, e fin dall'infanzia, si può esaltare il confronto tra interiorità ed esteriorità materiale e spirituale, celebrando libertà e autonomia d'azione nell'interdipendenza, nonché si possono gettare i semi per un raffronto fecondo tra diversità e culture, per la revisione concertata di una cultura sempre emergente, per una democrazia fattuale, giacché maturata nella prassi quotidiana dello scambio e della mediazione.

Concludendo, grazie a "Scarabò. Una città per educare" e grazie alla focalizzazione scientifica su Scarabò, si è avuto modo di comprendere che intavolare possibilità di "ri-unione" educativa e formativa può consentire di restituire centralità alla città e ai suoi spazi intesi come luoghi di interazione umanamente proficua e di azione condivisa, incidendo sul desiderio di una socialità educante. Da questa promozione dell'"agire in relazione" all'interno di centro storico vissuto occorre ripartire per ripensare la città secondo una prospettiva di corresponsabilità educativa che ha inevitabilmente da includere e considerare le opinioni dei bambini e delle bambine. Di quegli stessi bambini e bambine che, come B17D-19 (che brutto "nome" per un bambino), immaginano città con una sola porta d'ingresso perché le differenze si annullano nel momento in cui tutti passano da un'unica porta.

Da ultimo, è doveroso un ringraziamento alla dottoressa Laura Copparoni, ideatrice e regista del festival, per aver permesso la ricerca in parola, così come un sentito e profondo ringraziamento va agli studenti del corso di laurea in Scienze dell'educazione e della formazione dell'Università degli Studi di Macerata, senza i quali non avrei potuto condurre alcuna ricerca: Silvia Maffia, Alice Manoni, Francesca Merli, Giorgia Orsetti, Alessia Neroni, Federico Perri, Francesca Rendina, Beatrice Varani e Arianna Verri. 


\section{Riferimenti bibliografici}

Amadini M. (2003). Trans-formare lo spazio urbano: potenzialità pedagogiche della progettazione partecipata. In: Malavasi P., a cura di, Per abitare la Terra, un'educazione sostenibile. Milano: ISU.

Amadini M. (2012). Crescere nella città. Spazi, relazioni, processi partecipativi per educare l'infanzia. Brescia: La Scuola.

Bauman Z. (2003a). La solitudine del cittadino globale. Milano: Feltrinelli.

Bauman Z. (2003b). Voglia di comunità. Roma-Bari: Laterza.

Bazzicalupo L. (2013). Dispositivi e soggettivazioni. Milano-Udine: Mimesis.

Bertolini P. (1989). La città: un oggetto pedagogico?. In: Gennari M., a cura di, La città educante. Genova: Sagep.

Bertolini P. e Cardarello R., a cura di (1989). Da casa a scuola. Gli indicatori soggettivi della qualità di vita infantile. Firenze: La Nuova Italia.

Binanti L. (2007). Il bambino e la città. In: Limone P., a cura di, L'accoglienza del bambino nella città globale. Roma: Armando.

Borello C. (1989). La città come utopia educativa. In: Gennari M., a cura di, La città educante. Genova: Sagep.

Chicchi F. e Simone A. (2017). La società della prestazione. Roma: Ediesse.

Dardot P. e Laval C. (2013). La nuova ragione del mondo. Critica della ragione neoliberista. Roma: DeriveApprodi.

Dewey J. (1946). Liberalismo e azione sociale. Firenze: La Nuova Italia.

Dewey J. (1949). Democrazia e educazione. Firenze: La Nuova Italia.

Fernando J. y Morell S., coord. (1990). La Ciudad Educadora. Barcelona: Ayuntamiento de Barcelona.

Frabboni F. (1990). La integración escuela-territorio. In: Fernando J. y Morell S., coord. La Ciudad Educadora. Barcelona: Ayuntamiento de Barcelona.

Frabboni F. (1991). Un'aula grande come la mia città. In: Frabboni F. e Guerra I., a cura di, La città educativa. Verso un sistema formativo integrato. Bologna: Cappelli.

Gennari M. (1989). Architetture della città educante. In: Gennari M., a cura di, La città educante. Genova: Sagep.

Gennari M. (1995). Semantica della città e educazione. Venezia: Marsilio.

Harvey D. (2019). Geografia del dominio. Capitalismo e produzione dello spazio. Verona: Ombre Corte.

Hillman M., ed. (1993). Children, transport and the quality of life. London: Policy Studies Institute.

IAEC - International Association of Educating Cities, ed. (2008). Education and Urban Life: 20 Years of Educating Cities. Retrieved from: https://www.edcities.org/en/wp-content/uploads/sites/2/2015/02/Education-andUrban-Life.pdf [02/03/2020].

IAEC - International Association of Educating Cities, ed. (2009). City, Urban Planning and Education. Testo disponibile al sito: https:/www.edcities.org/en/wp-content/uploads/sites/2/2009/10/Monogr\%C3\%A1 fico-2009_en.pdf [02/03/2020].

Iori V. (2003). Spazio e tempo, fulcri educativi della pedagogia familiare. In: Pati L., a 
cura di, Ricerca pedagogica ed educazione familiare. Studi in onore di Norberto Galli. Milano: Vita e Pensiero.

Jordan L., Longworth N. and Osborne M. (2013). The rise and fall and rise again of learning cities. In: G.K. Zarifis e M. Gravani, eds., Challenging the 'European area of lifelong learning': A critical response. Dordrecht: Springer.

Kearns P. (2015). Learning city on the move. Australian Journal of Adult Learning, 55(1): 153-168.

Lefebvre H. (1970). Il diritto alla città. Padova: Marsilio.

Longworth N. (2007). Città che imparano. Come far diventare le città luoghi di apprendimento. Milano: Raffaello Cortina.

Longworth N. and Osborne M., eds. (2010a). Perspectives on Learning Cities and Regions. Policies, Practice and Participation. Leicester: Niace.

Longworth N. and Osborne M. (2010b). Six ages towards a learning region: a retrospective. European Journal of Education, 45(3): 368-406. DOI: 10.1111/j.14653435.2010.01436.x.

Lordon F. (2015). Capitalismo, desiderio e servitù. Antropologia delle passioni nel lavoro contemporaneo. Roma: DeriveApprodi.

Lorenzetto A. (1976). Lineamenti storici e teorici dell'educazione permanente. Roma: Studium.

Mencarelli M. (1986). Educazione permanente, democrazia, creazione culturale. In: Mencarelli M., a cura di, Educazione permanente e democrazia. Teramo: Giunti \& Lisciani.

Million A., Heinrich A.J., Coelen T., eds. (2017). Education, Space and Urban Planning. Education as a Component of the City. Cham: Springer.

OECD (1973). Recurrent Education: A Strategy for Lifelong Learning. Paris: OECD.

Ortega Esteban J. (1990). La idea de ciudad educadora a través de la historia. In: Fernando J. y Morell S., coord., La Ciudad Educadora. Barcelona: Ayuntamiento de Barcelona.

Osborne M., Kearns P. and Yang J. (2013). Learning cities: Developing inclusive, prosperous and sustainable urban communities. International Review of Education, 59(4): 409-423. DOI: 10.1007/s11159-013-9384-y.

Perucca A. (2007). Il gioco del convivere nella città globale. In: Limone P., a cura di, L'accoglienza del bambino nella città globale. Roma: Armando.

Piazza R. (2013). Learning city. Aspirazioni e ideali per le città del benessere. Roma: Aracne.

Richmond W.K. (1986). Democrazia e educazione continua. In: Mencarelli M., a cura di, Educazione permanente e democrazia. Teramo: Giunti \& Lisciani.

Save the Children (2018). Nuotare contro corrente. Povertà educativa e resilienza in Italia. Testo disponibile al sito: https://www.savethechildren.it/sites/default/files/files/uploads/pubblicazioni/nuotare-contro-corrente-poverta-educativa-e-resilienzaitalia.pdf [07/02/2020].

Scurati C. e Lombardi F.V. (1982). Il gioco. In: Scurati C. e Lombardi F.V., Pedagogia: termini e problemi. Milano: Scuola Vita.

Sennett R. (1992). La coscienza dell'occhio. Progetto e vita sociale nelle città. Milano: Feltrinelli. 
Sennett R. (2012). Insieme. Rituali, piaceri, politiche della collaborazione. Milano: Feltrinelli.

Spadafora G. (2011). L'educazione alla cittadinanza come problema epistemologico della democrazia. In: Corsi M. e Spadafora G., a cura di, Progetto generazioni. I giovani, il mondo, l'educazione. Napoli: Tecnodid.

Stenger J.R., ed. (2019). Learning cities in late antiquity. The local dimension of education. London-New York: Routledge.

Tizzi E.W. (1989). La città come spazio educativo e scolare. In: Gennari M., a cura di, La città educante. Genova: Sagep.

Tonucci F. (1996). La città dei bambini. Roma-Bari: Laterza.

Trilla Bernet J. (2005). La idea de ciudad educadora y escuela. Revista Educación $Y$ Ciudad, 7: 73-106.

Trisciuzzi L., Nuti L. e Viaggi M. (1993). Il territorio come libro di testo. Teramo: Giunti \& Lisciani.

UNESCO (2013). Beijing Declaration on Building Learning Cities. Lifelong Learning for All: Promoting Inclusion, Prosperity and Sustainability in Cities. Beijing: UNESCO. Text available at: https://www.dvv-international.de/fileadmin/files/beijing_declaration_en.pdf [07/02/2020].

Vercesi M. (2008). La mobilità autonoma dei bambini tra ricerca e interventi sul territorio. Milano: FrancoAngeli.

Yang J. (2012). An overview of building learning cities as a strategy for promoting lifelong learning. Journal of Adult and Continuing Education, 18(2): 97-113. DOI: 10.7227/JACE.18.2.8. 